\title{
Aspectos da atividade sexual precoce
}

\author{
Aspects of early sexual activity
}

A adolescência é caracterizada por acentuadas mudanças corporais e psíquicas devidas à crescente produção hormonal que influencia o desenvolvimento emocional e o comportamento. A Organização Mundial da Saúde (OMS) considera adolescência o período entre 10 e 19 anos de idade. No Brasil, o Estatuto da Criança e do Adolescente (Lei nº 8.069/1990) a define como a faixa etária entre 12 e 18 anos $^{1}$. As implicações jurídicas dessas definições são, às vezes, contrárias ao direito da população adolescente e podem interferir na prestação de cuidado à saúde física e emocional dessa população. O Ministério da Saúde do Brasil adverte que os adolescentes podem ser atendidos sozinhos, caso o desejem, e que devem ter a garantia de que as informações oferecidas nesse atendimento não serão repassadas aos seus pais e/ou responsáveis, bem como aos seus pares, sem concordância explícita² .

A expressão afetiva, emocional e erótica do adolescente reflete a expressão do seu comportamento sexual, que é construído no seio familiar desde a mais tenra idade. Nesse processo, os pais têm um papel fundamental, pois são eles os primeiros a auxiliar a criança a interpretar o ambiente em que vivem, e são a primeira referência para a criança que passa a mimetizar suas ações. A partir do convívio social, a aprendizagem da criança se amplia, incorporando diferentes parâmetros conceituais advindos do meio. O comportamento adotado resultará da interpretação desses diferentes ambientes, refletindo na expressão da sexualidade, ou seja, na forma com que o adolescente expressa a sua feminilidade, masculinidade ou ambas ${ }^{3}$. Um ambiente de violência física, emocional, sexual ou de negligência pode repercutir sobre o comportamento sexual do adolescente ${ }^{4,5}$. Por outro lado, um relacionamento assertivo com os pais tem reflexo positivo sobre sua sexualidade e saúde geral ${ }^{6}$.

O médico, especialmente o ginecologista, tem um papel importante na orientação sexual do adolescente visando sua saúde sexual ${ }^{7}$, mas a formação desse profissional para a abordagem da sexualidade é insipiente em nosso meio ${ }^{8}$. Isso contribui para que as orientações oferecidas por esse profissional sejam mais direcionadas para os riscos de doenças sexualmente transmissíveis (DSTs) e da gravidez não planejada em detrimento da abordagem do sexo como forma de prazer e informações sobre os riscos relacionados com a iniciação

Correspondência Lucia Alves da Silva Lara Avenida Bandeirantes, 3900 - Monte Alegre CEP: 14049-900 Ribeirão Preto (SP), Brasil Recebido $06 / 11 / 2014$
Ambulatório de Estudos em Sexualidade Humana, Departamento de Ginecologia e Obstetrícia da Faculdade de Medicina de Ribeirão Preto, Universidade de São Paulo - USP - Ribeirão Preto (SP), Brasil.

'Ambulatório de Estudos em Sexualidade Humana, Faculdade de Medicina de Ribeirão Preto, Universidade de São Paulo - USP Ribeirão Preto (SP), Brasil.

${ }_{2}^{2}$ Faculdade de Medicina, Universidade de São Paulo - USP - São Paulo (SP), Brasil,

Conflito de interesses: não há. 
sexual precoce ${ }^{9}$. Vale lembrar que as motivações para a iniciação sexual devem ser entendidas em uma perspectiva transcultural. Para sociedades que consideram a iniciação sexual como parte do processo de crescimento, a virgindade é interpretada como um estigma a ser vencido e a relação sexual se torna um acontecimento natural da vida ${ }^{10}$. Mas a primeira experiência sexual é mais do que um marco no desenvolvimento da mulher e pode ter implicações sobre seu bem-estar e sua saúde na vida adulta ${ }^{11}$.

Existe vasta literatura sobre a eficácia dos métodos de intervenção comportamental para a prevenção das DSTs, $\mathrm{HIV}^{12}$ e gravidez, mas praticamente não há estudos sobre as motivações que levam a adolescente a se engajar em relacionamento sexual. É possível que essa situação esteja associada à histórica repressão do direito ao prazer com o sexo, contraposto à noção de pureza pela virgindade ${ }^{13}$. A tendência atual é de iniciação sexual mais cedo, principalmente em países em desenvolvimento e quando não se conta com um programa de educação sexual consolidado nas escolas, como é o caso do Brasil, onde a iniciação sexual está ocorrendo em adolescentes com 13 anos ou menos ${ }^{14}$.

Embora o Código Penal Brasileiro presuma crime qualquer ato sexual com menores de 14 anos de idade, a sexarca (primeira relação sexual) é precoce entre as adolescentes, fato evidenciado em um estudo que investigou as características do comportamento sexual de 3.099 alunos de Goiânia, Goiás, com idade entre 13 e 15 anos $^{15}$. A Pesquisa Nacional de Saúde Escolar (PeNSE) avaliou o comportamento sexual de 60.973 adolescentes masculinos e femininos e evidenciou que cerca de um quarto deles tiveram relação sexual, a maioria com 13 anos ou menos, e cerca de $20 \%$ não usou proteção na última relação sexual ${ }^{14}$. Essa pesquisa, que foi realizada pelo Ministério da Saúde em parceria com o Instituto Brasileiro de Geografia e Estatística (IBGE), evidenciou que 26,5\% dos adolescentes, especialmente estudantes de escola pública e do sexo masculino, haviam tido pelo menos uma relação sexual, a maioria aos 13 anos, e já tinham tido até 3 parceiros. Os estudantes de escolas públicas tiveram mais informações sobre prevenção de gravidez e orientação sobre DST/ AIDS em relação aos que frequentaram a escola privada ${ }^{15}$. Nos países com maior nível educacional, os quais possuem mídia educativa e programas de ensino que incluem a educação sexual nas escolas, a iniciação sexual ocorre mais $\operatorname{tarde}^{16}$. Entre adolescentes americanos, a atividade sexual é rara antes dos 12 anos, ocorre em $30 \%$ dos adolescentes entre 15 e 16 anos e a maioria deles, entre 17 e 19 anos, é sexualmente ativa ${ }^{17}$.

As relações sexuais entre adolescentes são muitas vezes oportunistas e dependem do tempo que o jovem permanece ocioso ou sozinho em casa. Um estudo realizado em 6 escolas públicas americanas contou com 2.034 adolescentes e avaliou quando e onde os jovens tiveram suas relações sexuais e qual a relação entre o tempo sem supervisão e sexo, DSTs e uso de drogas. Nessa população, 73\% viviam com apenas um dos genitores, principalmente com a mãe $(52 \%)$ e $56 \%$ referiram estar em casa sem um adulto presente 4 ou mais horas por dia, depois da escola. Entre as meninas, 9\% tiveram sexarca antes dos 14 anos. Na população sexualmente ativa, 91\% haviam tido relação sexual em ambiente doméstico, incluindo a própria casa (37\%), a casa do seu parceiro (43\%), a casa de um amigo (12\%) e, geralmente, após a escola ${ }^{18}$. Nos dados da PeNSE de 2012 referentes aos adolescentes entre 13 e 15 anos, cerca de $25 \%$ tiveram relações sexuais e um quarto deles não usaram preservativo na última relação sexual. Os adolescentes que não tiveram orientação na escola sobre prevenção de gravidez apresentaram 1,41 vezes mais chance de ter relações sexuais e 1,87 mais chances de tê-las sem proteção ${ }^{19}$. Em 2010, um estudo com 17.371 alunos do ensino médio de escolas públicas e particulares de 27 capitais brasileiras evidenciou que cerca de um terço deles tiveram relações sexuais e quase a metade não usou preservativo, especialmente os de menor nível socioeconômico. O consumo excessivo de drogas ilícitas foi independentemente associado à relação sexual desprotegida ${ }^{20}$. Baixa escolaridade materna e mãe que trabalha aumentam a chance da adolescente adotar comportamento sexual de risco ${ }^{19}$. Quanto à etnia, meninas negras e as que experimentam cocaína são 4,88 vezes mais suscetíveis de engravidar em decorrência de comportamento sexual de risco ${ }^{21}$. Estudantes que não completaram o ensino médio têm maior número de parceiros sexuais em comparação com estudantes matriculados em programas de graduação de quatro anos ${ }^{22}$.

\section{Fatores associados à idade da sexarca}

Vários fatores contribuem para a sexarca precoce. Quanto maior o tempo em casa sem supervisão e sem ocupação, maior a possibilidade dos adolescentes se tornarem sexualmente ativos ${ }^{18}$. A falta de orientação na escola sobre prevenção da gravidez está associada à maior frequência de relações sexuais. No contexto familiar, ter pais separados ${ }^{23}$, viver apenas com um ou com nenhum dos pais e a baixa supervisão aumentam a frequência de relações sexuais ${ }^{14}$. Exposição precoce a cenas eróticas pode promover o comportamento sexual de risco, incentivar a busca por sensações sexuais precoces, múltiplos parceiros sexuais, uso inconsistente do preservativo, podendo também antecipar a iniciação sexual ${ }^{24}$. A idade precoce da sexarca está associada ainda à falta de emprego, ao pouco conhecimento sobre o HIV, ao uso de álcool e substâncias ilícitas ${ }^{25}$, ao abuso sexual na infância e a problemas familiares $^{26}$. Outro forte preditor de iniciação sexual precoce é a crença de que a maioria dos amigos já teve relação sexual $^{27}$. Em uma população de adolescentes brasileiros, 
a iniciação sexual precoce mostrou-se relacionada ao baixo nível socioeconômico, baixa escolaridade, ter pais divorciados, não praticar uma religião e uso de drogas ${ }^{28}$. Os jovens que pertencem a grupos específicos (tais como Hip-hop, Techno-scene, Metal, Punk, Skinheads) são mais propensos à iniciação sexual precoce, principalmente quando não possuem vínculo parental e não recebem monitoramento dos pais ${ }^{29}$.

A orientação sexual que a mãe oferece à adolescente é complexa e comumente mescla informações sobre saúde sexual e riscos das relações sexuais; aparentemente, o desejo sexual da filha é validado, porém controlado, no intuito de postergar a sexarca ${ }^{30}$. Um estudo avaliou o poder preditivo da percepção de adolescentes, masculinos e femininos, sobre as atitudes maternas a favor da abstinência sexual. Aproximadamente 10 mil adolescentes completaram duas entrevistas em suas casas com intervalo de um ano. Quanto maior a percepção dos adolescentes a respeito da desaprovação de suas mães sobre a iniciação sexual, maior foi a probabilidade de se envolverem em relações sexuais. As adolescentes satisfeitas com a relação com a mãe apresentaram menor probabilidade de iniciação sexual precoce e de gravidez, e maior probabilidade de fazer contracepção ${ }^{31}$. O Quadro 1 sumariza os fatores associados à maior frequência de sexarca precoce.

As implicações da sexarca precoce se referem ao maior risco de relações sexuais desprotegidas e uso inconsistente de métodos anticoncepcionais ${ }^{20}$ e, como consequência, aumento da incidência de gravidez não planejada e DSTs, risco aumentado para arrependimento, depressão e ideação suicida ${ }^{32}$. Diante disso, fica evidente a necessidade de medidas que visam postergar a iniciação sexual ${ }^{33}$, reduzir os relacionamentos sexuais transitórios com múltiplos parceiros e a promoção do sexo seguro pelo uso consistente do preservativo. Essas iniciativas devem ser complementadas com medidas de prevenção da violência sexual (abuso sexual de crianças, sexo não consentido) e da discriminação de gênero ${ }^{34}$.

Quadro 1. Fatores associados ao risco de sexarca precoce

\begin{tabular}{|ll|}
\hline Estudos (Referências) & \multicolumn{1}{c|}{ Fafores que abreviam a idade da sexarca } \\
\hline 26 & Influência do parceiro, pulsão sexual, história de abuso sexual, a relação sexual é uma prova de amor \\
$35-37$ & Cor negra, ser filha de mãe adolescente \\
29 & Falta de monitoramento dos pais \\
$14,23,28$ & Pais separados, baixo nivel escolar, viver com apenas um genitor ou sem os genitores \\
$38-40$ & Baixa autoestima, insegurança, programas espećficos de televisão, desconhecimento sobre DSTs/HIV, influência social e do meio em que vive, relacionamento com os pais negligente \\
14 & Falta de educação sexual na escola \\
28,37 & Baixa escolaridade, baixo rendimento escolar \\
41 & Estímulo sexual precoce, insistência do parceiro \\
28 & Baixa condição socioeconômica \\
$25,37,42$ & Falta de emprego, uso de drogas eálcool \\
28 & Não praticar religião \\
27 & Amigos que tiveram relaç̦õo sexual \\
\hline
\end{tabular}

\section{Referências}

1. Digiácomo M, Digiácomo IA. ECA: Estatuto da Criança e do Adolescente anotado e interpretado. São Paulo: FTD; 2010.

2. Brasil. Ministério da Saúde. Secretaria de Atenção à Saúde. Saúde integral de adolescentes e jovens: orientações para a organização de serviços de saúde. Brasília (DF): Editora do Ministério da Saúde; 2005.

3. van de Bongardt D, Reitz E, Sandfort T, Deković M. A meta-analysis of the relations between three types of peer norms and adolescent sexual behavior. Pers Soc Psychol Rev. 2014. pii: 1088868314544223. [Epub ahead of print]

4. Hulland EN, Brown JL, Swartzendruber AL, Sales JM, Rose ES, DiClemente $R$. The association between stress, coping, and sexual risk behaviors over 24 months among African-American female adolescents. Psychol Health Med. 2014:1-14. [Epub ahead of print]
5. Fowler PJ, Motley D, Zhang J, Rolls-Reutz J, Landsverk J. Adolescent maltreatment in the child welfare system and developmental patterns of sexual risk behaviors. Child Maltreat. 2015. doi: 10.1177/1077559514548701. [Epub ahead of print]

6. Rickert VI, Gilbert AL, Aalsma MC. Proactive parents are assets to the health and well-being of teens. J Pediatr. 2014;164(6):1390-5.

7. Yulevitch A, Czamanski-Cohen J, Segal D, Ben-Zion I, Kushnir T. The vagina dialogues: genital self-image and communication with physicians about sexual dysfunction and dissatisfaction among Jewish patients in a women's health clinic in southern Israel. J Sex Med. 2013;10(12):3059-68.

8. Lara LA. [Sexuality, sexual health and sexual medicine: current overview]. Rev Bras Ginecol Obstet. 2009;31(12):583-5. Portuguese. 
9. McCurdy SA. Willingness to provide behavioral health recommendations: a cross-sectional study of entering medical students. BMC Med Educ. 2012;12:28.

10. Erickson PI, Badiane L, Singer M. The social context and meaning of virginity loss among African American and Puerto Rican young adults in Hartford. Med Anthropol Q. 2013;27(3):313-29.

11. Smith CV, Shaffer M. Gone but not forgotten: virginity loss and current sexual satisfaction. J Sex Marital Ther. 2013;39(2):96-111.

12. Protogerou $C$, Johnson BT. Factors underlying the success of behavioral HIV-prevention interventions for adolescents: a meta-review. AIDS Behav. 2014;18(10):1847-63.

13. Molla M, Berhane $Y$, Lindtiørn B. Traditional values of virginity and sexual behaviour in rural Ethiopian youth: results from a cross-sectional study. BMC Public Health. 2008;8:9.

14. Oliveira-Campos M, Giatti L, Malta D, Barreto SM. Contextual factors associated with sexual behavior among Brazilian adolescents. Ann Epidemiol. 2013;23(10):629-35.

15. Sasaki RS, de Souza MM, Leles CR, Malta DC, Sardinha LM, Freire MC. Sexual behavior of school-aged adolescents in the city of Goiânia, Goiás. Rev Bras Epidemiol. 2014;17(Suppl 1):172-82.

16. Dijanić T, Kozul K, Miskulin M, Medić A, Jurcev-Savicević A, Burazin J. Sexual behaviour and condom use as a protection against sexually transmitted infections in student population. Coll Antropol. 2014;38(1):31-7.

17. Finer $L B$, Philbin $J M$. Sexual initiation, contraceptive use, and pregnancy among young adolescents. Pediatrics. 2013;131(5):886-91.

18. Cohen DA, Farley TA, Taylor SN, Martin DH, Schuster MA. When and where do youths have sex? The potential role of adult supervision. Pediatrics. 2002;110(6):e66.

19. Oliveira-Campos M, Nunes ML, Madeira FC, Santos MG, Bregmann SR, Malta DC, et al. Sexual behavior among Brazilian adolescents, National Adolescent School-based Health Survey (PeNSE 2012). Rev Bras Epidemiol. 2014;17(Suppl 1):116-30.

20. Sanchez ZM, Nappo SA, Cruz JI, Carlini EA, Carlini CM, Martins SS. Sexual behavior among high school students in Brazil: alcohol consumption and legal and illegal drug use associated with unprotected sex. Clinics (Sao Paulo). 2013;68(4):489-94.

21. Rome ES, Rybicki LA, Durant RH. Pregnancy and other risk behaviors among adolescent girls in Ohio. J Adolesc Health. 1998;22(1):50-5.

22. Lyons HA, Manning WD, Longmore MA, Giordano PC. Gender and casual sexual activity from adolescence to emerging adulthood: social and life course correlates. J Sex Res. 2014:1-15. [Epub ahead of print]

23. Tsitsika A, Andrie E, Deligeoroglou E, Tzavara C Sakou I, Greydanus D, et al. Experiencing sexuality in youth living in Greece: contraceptive practices, risk taking, and psychosocial status. J Pediatr Adolesc Gynecol. 2014;27(4):232-9.

24. O'Hara RE, Gibbons FX, Gerrard M, Li Z, Sargent JD. Greater exposure to sexual content in popular movies predicts earlier sexual debut and increased sexual risk taking. Psychol Sci. 2012;23(9):984-93.

25. Tilahun $M$, Ayele $G$. Factors associated with age at first sexual initiation among youths in Gamo Gofa, south west Ethiopia: a cross sectional study. BMC Public Health. 2013;13:622.
26. Nik Farid ND, Che' Rus S, Dahlui M, Al-Sadat N. Determinants of sexual intercourse initiation among incarcerated adolescents: a mixed-method study. Singapore Med J. 2013;54(12):695-701.

27. Kinsman SB, Romer D, Furstenberg FF, Schwarz DF. Early sexual initiation: the role of peer norms. Pediatrics. 1998; 102(5): $1185-92$.

28. Hugo TD, Maier VT, Jansen K, Rodrigues CE, Cruzeiro AL, Ores LC, et al. [Factors associated with age at first intercourse: a population-based study]. Cad Saúde Pública. $2011 ; 27(11): 2207-14$. Portuguese.

29. Bobakova D, Geckova AM, Klein D, van Dijk JP, Reijneveld SA. Protective and risk factors of early sexual initiation in youth subcultures. Eur J Contracept Reprod Health Care. 2013;1 8(4):242-50.

30. Nelson E, Edmonds A, Ballesteros M, Encalada Soto D, Rodriguez $O$. The unintended consequences of sex education: an ethnography of a development intervention in Latin America. Anthropol Med. 2014;21(2):189-201.

31. Dittus PJ, Jaccard J. Adolescents' perceptions of maternal disapproval of sex: relationship to sexual outcomes. J Adolesc Health. 2000;26(4):268-78.

32. Kim DS, Kim HS. Early initiation of alcohol drinking, cigarette smoking, and sexual intercourse linked to suicidal ideation and attempts: findings from the 2006 Korean Youth Risk Behavior Survey. Yonsei Med J. 2010;51(1):18-26.

33. Postrado LT, Nicholson HJ. Effectiveness in delaying the initiation of sexual intercourse of girls aged 12-14. Two components of the Girls Incorporated Preventing Adolescent Pregnancy Program. Youth Soc. 1992;23(3):356-79.

34. Allen CF, Edwards P, Gennari F, Francis C, Caffe S, Boisson E, et al. Evidence on delay in sexual initiation, multiple partnerships and condom use among young people: review of Caribbean HIV behavioural studies. West Indian Med J. 2013;62(4):292-8.

35. Coker AL, Richter DL, Valois RF, McKeown RE, Garrison CZ, Vincent ML. Correlates and consequences of early initiation of sexual intercourse. J Sch Health. 1994;64(9):372-7.

36. Handler A. The correlates of the initiation of sexual intercourse among young urban black females. J Youth Adolesc. 1990; 19(2): 159-70.

37. Santelli JS, Kaiser J, Hirsch L, Radosh A, Simkin L, Middlestadt S. Initiation of sexual intercourse among middle school adolescents: the influence of psychosocial factors. J Adolesc Health. 2004;34(3):200-8.

38. Peterson JL, Moore KA, Furstenberg FF Jr. Television viewing and early initiation of sexual intercourse: is there a link? J Homosex. 1991;21(1-2):93-118.

39. Tenkorang EY, Maticka-Tyndale E. Factors influencing the timing of first sexual intercourse among young people in Nyanza, Kenya. Int Fam Plan Perspect. 2008;34(4): 177-88.

40. Price MN, Hyde JS. When two isn't better than one: predictors of early sexual activity in adolescence using a cumulative risk model. J Youth Adolesc. 2009;38(8):1059-71.

41. Osorio A, López-del Burgo C, Carlos S, Ruiz-Canela M, Delgado $M$, de Irala J. First sexual intercourse and subsequent regret in three developing countries. J Adolesc Health. 2012;50(3):271-8.

42. Sales JM, Monahan JL, Brooks C, DiClemente RJ, Rose E, Samp JA. Differences in sexual risk behaviors between lower and higher frequency alcohol-using African-American adolescent females. Curr HIV Res. 2014;12(4):276-81. 